\title{
POVERTY AND LABOUR MARKET MARKERS OF HIV POSITIVE HOUSEHOLDS IN SOUTH AFRICA: AN EXPLORATORY METHODOLOGICAL ANALYSIS
}

\author{
NAJMA SHAIKH AND HAROON BHORAT ${ }^{1}$
}

\begin{abstract}
This study, through an exploratory, but promising methodology, provides a tentative analysis of the relationship between HIV, poverty and labour markets. This was undertaken through the use of the October Household Survey of 1999 and the Antenatal Clinic Survey of 1998. It is clear from the paper that the relationship between poverty, labour markets and HIV is not homogeneous but multi-dimensional in character. The analysis examines these interrelationships at both the household and individual level. The preliminary key finding from the analysis is that imputed HIV positive women come from poorer households than imputed negative women, but this observed difference is small. It is suggested that the analysis be repeated using larger samples of data, such as that now available from Census 2001
\end{abstract}

While the HIV epidemic continues to mature in South Africa, showing no signs of plateauing, it is clear that South Africa(SA) stands at the brink of an AIDS crisis. Some of the salient features of the AIDS pandemic are understood in terms of the demographic profile of those who are inflicted, namely that HIV/AIDS tends to strike young, heterosexual and sexually active adults (DOH, 1998; DOH, 2000). However, the economic implications of the pandemic remain murky at best, with a limited understanding of how HIV related mortality and morbidity affect productivity, factor returns, employment, income distribution, savings rates, consumption patterns and other economic variables.

In addition, there is very little empirically robust information on the social and welfare correlates of the pandemic. Understanding these economic implications is critical in the post-apartheid period. While there has been debate about the impact of HIV/AIDS on poverty, the link between HIV/AIDS prevalence and the various determinants of poverty and labour market outcomes has not been rigorously dealt with in the South African literature. One of the key obstacles to understanding these issues is the absence of hard data on HIV status and its association with poverty, inequality and labour markets. The purpose of this paper is therefore to use existing household survey data to provide a tentative window into the households that an imputed sub-set of HIV/AIDS sufferers and their dependents emanate from. Hence, the core focus of this paper is to present a novel methodology that may be utilized on existing sources of information to analyse the features of the HIV/AIDS epidemic at the household level.

1 Epidemiology Consultant, Provincial Administration of the Western Cape (PAWC) and Director, Development Policy Research Unit (DPRU), School of Economics, University of Cape Town respectively. The authors would like to thank Stephen Dike, Elizabeth Ninan and Hilary Southall for their invaluable comments and suggestions. Our thanks must also go to officials from the Department of Health, in particular Pakiso Netshidzivhani, Rika du Plessis and Rose Malumba, who graciously facilitated access to the relevant data. 


\section{METHODOLOGY}

The paper is designed to develop a method of analysing the household characteristics associated with HIV status using currently available data. There is a dearth of robust household level data linking HIV status to socio-economic and demographic factors. Household surveys are expensive and labour intensive, and are particularly subject to bias in ascertaining HIV status, given factors associated with stigma and confidentiality. Within these constraints this paper utilises the October Household Survey of 1999 (OHS99), a nationally representative household survey produced by Statistics South Africa (Stats SA), in conjunction with published statistics from the antenatal clinic (ANC) data of the national Department of Health.

\section{(a) Data Sources}

The annual national HIV antenatal sentinel surveys which are conducted by the national Department of Health remain the primary source of data in SA reflecting the trends and magnitude of HIV infection amongst young, sexually active, heterosexual adults (Shaikh and Abdullah, 2002). The data derived from the ANC surveys form the primary source data for modelling the growth of the epidemic and its sociodemographic impact, as well as for planning, monitoring and evaluating HIV strategies and programmes (DOH, 2000; WHO, 2000).

Each year, during the month of October, pregnant women attending public sector clinics are anonymously examined for HIV. This group is targetted because they are considered to be representative of the sexually active, heterosexual members of the female population and are accessible for blood testing as this forms an integral part of the routine antenatal clinical care at the first visit. The primary aim of the survey is to estimate HIV sero-prevalence at the provincial and national level. A two-stage random cluster sample using the Probability Proportional to Size (PPS) method is used, weighting for provincial populations (DOH, 1999). This allows for a self-weighted sample, where each attendee has an equal chance of being selected for the survey. A total of 15089 specimens in 1998 were collected country wide. The same sites (sentinel sites) are used each year for the survey as this allows tracking of the epidemic over time.

After the routine pregnancy education session and following verbal consent, blood specimens are collected, transported and processed according to standard operational procedures outlined in the national protocol, and according to the WHO guidelines for unlinked sentinel surveys (WHO, 2000; Nicoll et al., 2000).

For the OHS99, as for all previous October national household surveys, a sample of 30000 households was drawn in 3000 Enumerator Areas (EAs), and exactly 10 households per EA. A two-stage sampling procedure was applied and the sample was stratified by province and location, clustered and selected to meet the requirements of probability sampling. The sample was based on the 1996 Population Census enumerator areas and the estimated number of households from the 1996 Population Census. Within each explicit stratum the EAs were stratified by simply arranging them in geographical order by District Council, Magisterial District and, within the magisterial district, by average household income (for formal urban areas and hostels) or EA (Statistics South Africa, 1999). The allocated number of EAs was systematically selected with Probability Proportional to Size in each stratum. The measure of size was the estimated number of households in each EA.

The questionnaire had seven sections, which covered a range of issues including 
standard person and household characteristics, details of births and deaths within the household, household income and expenditure, and fairly detailed labour market information. These particular issues are explored through the rest of this paper.

\section{(b) The Matching Process}

The broad approach in this exercise involved linking the data from the ANC survey of 1998 to the OHS99 data set, by randomly allocating ${ }^{2}$ proportions to a sub-sample of women from the OHS99 who used public sector clinics and were pregnant in the previous year. The ANC survey runs in October, the same month as the OHS, and hence seasonal variation between the two data sets was not a problem. The OHS99 has data on which women in this age range, gave birth in the last year, and hence, had a high likelihood of having been pregnant in 19983. This sample of 1998 pregnant women from the OHS99 therefore provided the first initial match to the ANC98 data. Through this sample of pregnant women in the OHS99, we attempted to determine the labour market and poverty correlates of HIV/AIDS sufferers and the households they reside in.

Since one cannot assume that all pregnant women derived in the OHS99 sample were $\mathrm{HIV}+$, the ANC98 published results were critical for providing these imputed prevalence figures. The distribution of $\mathrm{HIV}+$ pregnant women were calculated by province, age and education levels. Hence, in theory, one is able to derive from the ANC98 data a three-dimensional matrix containing the proportion of HIV+ women as a percentage of the total number of women in each cell of the matrix. We can define the matrix for example as $\rho_{\mathrm{ijk}}$ where $\rho$ is the share of women in the ANC98 sample who are reported as HIV + according to province $i$, age $j$ and educational category $k$.

The ANC98 data could then be aligned in a manner where each of the individuals in the sample was coded according to the above procedure. Hence, we can arrive at values of $\rho_{\mathrm{ijk}}$ for each of the women in the sample. Hence, with the ANC98 data, we can derive estimates of $\rho_{\mathrm{ijk}}$ that would yield estimates of the percentage of women who are $\mathrm{HIV}+$ according to all combinations of the categories $i, j$ and $k$.

These values for $\rho_{\mathrm{ijk}}$ can then be used to impute the HIV status of pregnant women in the OHS99 dataset, given that we had the values for $\rho_{\mathrm{ijk}}$ for each possible combination of province, age and education categories from the ANC98 data. In terms of the OHS99 data then, we would use the sample of women who reported giving birth in the last year, and attended a public clinic, and re-categorise the age and education variables, to match up with the chosen educational categories and age cohorts from the ANC98 data. Simply put, the $\rho_{\mathrm{ijk}}$ values are those we take from the ANC98 dataset and then imputed into the OHS99 sample. For example then, if the ANC98 data suggested that of those women in province 1 , age category 1 and education cohort 1, 56 per cent were $\mathrm{HIV}+$, this is the proportion we randomly impute as HIV+ into OHS99, should

2 Given that the random process is subject to instability, 35 replications of the random assignment according to the ANC rates were done and the mean of the variable under consideration was reported.

3 The match though is an inexact one: from the ANC data in October 1998, it implies that a woman would have become pregnant in the period February 1998 to September 1998, and hence would have given birth in the period November 1998 to June 1999. While there is an overlap, the period of birth from about July 1999 to September 1999 is not captured in the ANC data set for 1998 . 
an individual fit into that category.

\section{RESULTS FROM THE IMPUTATION EXERCISE}

The OHS99 sample firstly needed to be cleaned of any obvious anomalies ${ }^{4}$. Secondly, one of the key additions required here was to ensure that the age profile of these women matched with those in the ANC data. Hence, we retained all pregnant women between the ages of 12 and 49 who reported attending a public sector clinic in the last year. Ultimately then, the final sample to be utilized for our matching process was 1659 pregnant women between the ages of 12 and 49 who reported attending a public sector clinic.

Given that this is an extremely small sample, the results reported must be treated with caution. The smallness of the sample is a function primarily of the initial sample size of the OHS, where 30000 household and just over 100000 individuals were surveyed. The strength of the paper lies in the fairly rigorous methodological approach proposed for accessing socio-economic information on the pandemic, in the absence of direct survey data. The paper should be seen in this light: that while we report on the labour market and poverty markers of HIV/AIDS, we accept the limitations inherent in the reported results due to the small sample size ${ }^{5}$.

Having imputed an HIV status onto the 1659 women to be used in our analysis according to their age, education provincial categories, it became clear that the small sample size would militate against deriving too large a number of cells. We eventually decided on a matrix with three age categories and two education categories, excluding the province variable ${ }^{6}$. The results are shown in Table 1.

Table 1. Number OHS99 sample of pregnant women attending public health clinics

\begin{tabular}{llll}
\hline Age & Less than Matric & Matric + & Total \\
$12-19$ & 260 & 17 & 277 \\
Per cent of total & 15.67 & 1.02 & 16.70 \\
$20-29$ & 591 & 238 & 829 \\
Per cent of total & 35.62 & 14.35 & 49.97 \\
$30-49$ & 464 & 89 & 553 \\
Per cent of total & 27.97 & 5.36 & 33.33 \\
Total & 1315 & 344 & 1659 \\
Per cent of Total & 79.26 & 20.74 & 100 \\
\hline
\end{tabular}

Source: OHS99 and authors' own calculations

The biggest single group in the sample were women between the ages of 20 and 29 who reported having less than a matric as their highest educational qualification. These women formed about 36 per cent of the sample and numbered 591. The second-largest group also had less than a matric, but were between the ages of 30 and 49. Indeed,

\footnotetext{
${ }^{4}$ For example, the sample of women who reported bearing a child in the last year, contained at least 10 women over the age of 60 and one man!

${ }^{5}$ One obvious way around this problem would have been to use census data. However, using the Census 1996 data is problematic given that it is accepted that the ANC for 1995 is not reliable. In turn, the Census 2001 results were not available at the time of writing.

${ }^{6}$ Initially, we had started out with a $3 \times 3$ matrix that had three instead of two education variables. However the resulting prevalence was so similar for the matric + and tertiary categories, that it was decided to collapse these two categories into one. The prevalence figures for this are available from the authors on request.
} 
these two categories formed just under 65 per cent of the entire sample of pregnant women. The dominating characteristic in this sample was the level of educational attainment, where close to 80 per cent of the women had not completed a matric.

The next step was to determine the prevalence figures according to these categories, as derived from the antenatal clinic data for 1998. The prevalence figures were calculated from an underlying sample of 15089 women, of whom 3170 tested positive for HIV/AIDS.

The data revealed a national prevalence figure of approximately 23 per cent for the full sample. The highest prevalence was among the 20-29 age group, who also formed the largest sample of the women in the OHS dataset. The lowest prevalence was among women with less than a matric and between the ages of 30 and 49. These figures provide very early evidence that the pandemic has a strong youthful dimension and indeed does not seem to vary greatly across educational categories.

Table 2. HIV + Prevalence from ANC98 by education and age categories

\begin{tabular}{llll}
\hline Category & Less than Matric & Matric + & Total \\
$12-19$ & $18.05(0.013)$ & $25.10(0.112)$ & $19.04(0.011)$ \\
$20-29$ & $23.94(0.009)$ & $19.71(0.029)$ & $24.13(0.008)$ \\
$30-49$ & $15.59(0.007)$ & $17.11(0.029)$ & $15.35(0.007)$ \\
Total & $20.68(0.008)$ & $19.40(0.025)$ & $22.82(0.007)$ \\
\hline
\end{tabular}

Source: ANC 98 and authors' own calculations

Note: Standard errors are in parentheses, and are corrected for frequency weights, the primary sampling unit and sampling stratification.

Given the above two Tables, we then aligned the data sets for the matching process. Specifically, the procedure was to randomly assign an HIV status to pregnant women in the OHS sample, according to the distributional parameters outlined in the ANC prevalence figures. For example, the prevalence among women in the age category 1219 with less than a matric was derived from the ANC98 as 18.05 per cent. Within the OHS data we then randomly assigned an HIV positive status to 18.05 per cent of all women in this particular cell. This process was repeated across all the categories, to arrive at imputed HIV statuses for the pregnant women in the OHS sample \{Table 3). However, as alluded to earlier, the random process itself is subject to instability, in that different individuals are likely to be assigned an imputed HIV status with each random allocation undertaken. This is no doubt exacerbated by the small sample size in the OHS survey. In dealing with this instability, we undertook 35 replications of the random assignment according to the ANC rates.

Table 3. Imputed HIV Prevalence for Pregnant Public Clinic

\begin{tabular}{llll}
\hline Age & Less than Matric & Matric + & Total \\
$12-19$ & $18.08(0.023)$ & $23.53(0.103)$ & $18.41(0.023)$ \\
$20-29$ & $23.86(0.018)$ & $19.75(0.026)$ & $22.68(0.015)$ \\
$30-49$ & $15.52(0.017)$ & $16.85(0.040)$ & $15.73(0.015)$ \\
Total & $19.77(0.011)$ & $19.19(0.021)$ & $19.65(0.009)$ \\
\hline
\end{tabular}

Source: OHS99 and ANC98 and authors' own calculations

Note: Standard errors are in parentheses, and are corrected for by frequency weights, primary sampling unit and sampling stratification.

Although the overall imputed HIV prevalence in the analysis group was lower (19.6 per cent) than the prevalence estimated from the ANC data (22.1 per cent), age-specific 
figures showed consistency in ranking with the ANC dataset in that the highest prevalence was observed in the 20-29 age group

Given that we randomly distributed HIV status across a sample size that is smaller than that contained in the ANC data, we expected some variation in the resulting imputed prevalence figures from the true estimates. The discrepancy in the estimates was not too large to render the imputed HIV status figures unusable.

\section{SOCIO-ECONOMIC CORRELATES OF IMPUTED HIV STATUS}

One useful analytical tool in trying to determine some of the socio-economic factors associated with HIV prevalence, is to segment the descriptive statistics into the household and individual characteristics that are correlated with imputed HIV status. Hence, we will attempt, in the sections that follow, to interrogate the nature of the households that these women reside in, allowing for the fact that this is a relatively small sample. Secondly, the individual characteristics of these women are clearly important in a variety of different ways, as they may further illuminate on the nature of the pandemic

\section{(a) Household Characteristics By Imputed HIV Status}

Mindful of the limitations inherent in a sample that it is relatively small, we nevertheless initiate the empirical overview in this section, by examining the spatial distribution of the epidemic. About 56 per cent (44 per cent) of all women in the group lived in urban (rural) areas. According to imputed HIV status, there was a larger proportion of imputed HIV positive women in urban areas, compared with imputed HIV negative women - 56 per cent compared with 54 per cent. We report in the Table below the prevalence figures for women residing in rural and urban households. The evidence suggests that the prevalence in urban areas is marginally higher.

\section{Table 4. Imputed HIV Prevalence for Pregnant Clinic Attendees, By Location}

\begin{tabular}{lllll}
\hline Location & Estimate & Std. Err. & $\mathbf{9 5 \%}$ Conf. Interval & \\
Rural & 19.64 & 1.46 & 16.763 & 22.508 \\
Urban & 19.66 & 1.31 & 17.093 & 22.231 \\
Total & 19.65 & 0.98 & 17.736 & 21.564 \\
\hline
\end{tabular}

Source: OHS99 and authors' own calculations

If we assume however, that the small sample size may drive the insignificance in the results, and take the higher urban prevalence as robust, these results would be consistent with other studies within Africa, where higher rates of HIV + have been observed in urban relative to rural settings (Alfowesco 1995; Topouzis 1998). This is attributed to a range of factors such as population density, rapid urbanization, unstable families and migrancy, all of which are reported to lead to higher HIV rates (Quinn, 1996).

One key welfare aspect of household attachment amongst individuals is the level of household income that infected individuals may have access to. Within the context of this paper, it was therefore important to examine the distribution of household income according to imputed HIV status. The income data within the OHS was only provided according to categories, and hence point estimates were not possible to derive ${ }^{7}$. The

\footnotetext{
7 Measures of poverty and inequality, typically the headcount index, the poverty gap measure and Gini coefficient cannot be derived from data that is in ranges rather than point estimates of
} 
best presentation of the data then, was to examine the distribution of household income across the different categories by imputed HIV status. We present the cumulative distribution of household monthly income across the two categories in Fig. 1 below.

The two distributions suggest that there is first-order dominance. This means that the distribution of household income for imputed HIV positive women lies above that of the distribution for imputed HIV negative women for each income category provided. It is only at the tail-ends of the distributions that there appears to be some convergence. This provisional evidence seems to suggest that women with an imputed HIV positive status emanate from poorer households.

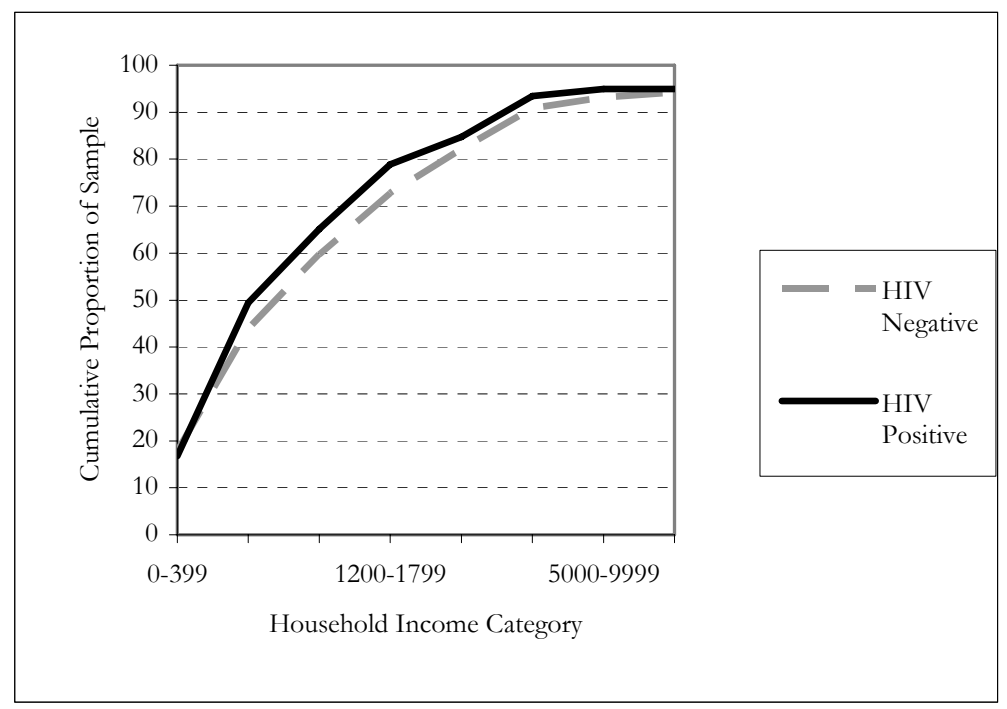

Figure 1. Cumulative Density Function of Total Monthly Income by Imputed HIV

Status (OHS99)

Source: OHS99 and authors' own calculations

More specifically, the proportion of households with imputed HIV negative women, earning a monthly income of R799 or less is about 44 per cent. The corresponding figure for households with imputed HIV positive women is approximately 49 per cent. Further up the distribution, the percentage of households earning R1199 per month or less is 60 per cent for imputed HIV negative households and 69 per cent for imputed HIV positive households. Put differently, if we impose an arbitrary household poverty line of say R1799 per month, then the headcount index for imputed HIV negative households would be 73 per cent and the measure for imputed HIV positive households would be 79 per cent. Within the constraints of the data, this crude measure of poverty, provisionally suggests differentials in the levels of household income across imputed HIV status in this sample of pregnant women. Apart from small sample size constraints, one of the critical caveats to this first result, is that as we do not have point estimates of monthly household income, it is not possible to accurately assess whether these two distributions are significantly different from each other. We therefore

income for each household Comparing the poverty and inequality levels of households by imputed HIV status would of be very illuminating. 
interrogated the data further, in an attempt to explain and validate these initial findings through testing for significant differences in the sub-section below.

(b) Measures of Absolute and Relative Poverty Differences

Testing for significant differences in the forms of the above cumulative distribution functions was not possible, without some sense of a continuous income variable. Unfortunately, as a result of the data, we only had the income variable reported according to pre-specified categories. These categories, as Fig. 1 above illustrates, range from monthly household income of between 0 and R399 to R10000 or more. In an attempt to provide some semblance of a point measure, we have created point estimates within the categories. This was done by simply placing all those households within a category range at the mean of the range. Hence, all households in the 0-399 range, were placed at a monthly income value of R199.5. We therefore derived a sample of household income for imputed HIV positive and negative women that effectively allowed us to provide proxies of poverty measurement, and furthermore test for differences.

In order to measure poverty levels in this sample, according to monthly income, we utilized the general class of poverty measures first proposed by Foster, Greer and Thorbecke (1984), and known more widely as the 'FGT' measures of poverty. The FGT index of poverty measures can be represented in general form as:

$$
P_{\alpha}=\frac{1}{n} \sum_{i=1}^{n}\left(\frac{z-y_{i}}{z}\right)^{\alpha} \mid\left(y_{i} \leq z\right)
$$

where $n$ is the sample size, $z$ is the chosen poverty line, and $y_{i}$ is the standard of living indicator of agent $i$. The parameter $\alpha$ measures how sensitive the index is to transfers between the poor units. Note that the index is conditional on the agent's income, $y_{i}$, being below the designated poverty line, ₹. The headcount index is generated when $\alpha=0$, and in this case equation (1) is then simply the share of agents below the poverty line. The poverty gap measure (PG) is generated when $\alpha=1$, and therefore for a given poverty line $\%$, is presented as:

$P_{1}=\frac{1}{n} \sum_{i=1}^{n}\left(\frac{z-y_{i}}{z}\right)\left(y_{i} \leq z\right)$

The PG measure represents a direct measure of agents' incomes relative to the poverty line. A first advantage of the FGT index, is its additive decomposability, which allows for sub-group poverty measures to be summed to form a society-wide measure without loss of generality. More directly though, the PG allows a more nuance assessment of relative poverty - something that the standard headcount index cannot provide. Using this measure, the headcount and poverty gap measures for imputed HIV positive and HIV negative households were derived as a direct complement to the cumulative distribution functions above (Table 5). We set a poverty line (z) of R1499.5, which is the midpoint of the $4^{\text {th }}$ income category, 1200 to 1799 Rands per month.

At the poverty line of R1499.5 per month, 66 per cent of households with imputed HIV negative women were poor (Table 5). In addition, these poor households were earning incomes that placed them, on average, 43 per cent below the stipulated poverty line. The latter is our measure of relative poverty, captured through this poverty gap 
estimate. In comparison, households with imputed HIV positive women yielded a headcount estimate of 70 per cent, and a poverty gap of 45 per cent. Hence, the data provisionally suggests that imputed HIV positive women may be residing in poorer households than imputed HIV negative women.

Table 5. Headcount and Poverty Gap Measures for Imputed HIV Status Households ${ }^{8}$

\begin{tabular}{lllll}
\hline Poverty Measure & Estimate & Std. Error & Confidence Interval & \\
HIV Negative & & & & 0.6850 \\
Headcount Index & 0.66 & 0.014 & 0.6290 & 0.4466 \\
Poverty Gap & 0.43 & 0.011 & 0.4045 & 0.7581 \\
HIV Positive & & & & 0.48499 \\
Headcount Index & 0.70 & 0.027 & 0.4082 & -0.99 \\
Poverty Gap & 0.45 & 0.020 & Poverty Gap & \\
t-Statistics & Headcount & -1.52 & & \\
\hline
\end{tabular}

Source: OHS99 and authors' own calculations

Note that this statement is true for both the absolute measure of poverty (the headcount index) and the relative measure of poverty (the poverty gap). The results of the $t$-statistic reveal that for both the headcount and the poverty gap measure, the estimates for imputed HIV positive and negative women are not statistically different from each other. However, it is possible that this is the result of a small sample size, rather than representing a robust acceptance of the null hypothesis that these poverty estimates are the same across both cohorts of women. At a minimum though, the results do point to the fact that household vulnerability may be critical to understanding the burden of the epidemic.

\section{(c) Attachment to Wage Earners and the Quality of Support}

The labour market remains probably the most important means for households to generate income on a regular basis. More broadly, the availability of wage income through formal or informal employment determines the nature of household poverty and inequality in a society; and in all economies of course, the labour market remains the key avenue for this income accumulation. Simply put, the labour market remains the filter through which the long-run distributional and poverty outcomes of a society are shaped. It was therefore necessary to examine the degree to which imputed HIV positive and negative women had access to employed individuals within the household. As a first step in gauging how effectively labour market activity within these respective households may be translating into lower levels of vulnerability, we estimated the number of employed individuals residing within each of the households.

The distribution of these households according to the number of employed individuals within each household is shown in Table 69 . Firstly, in both categories, more than half of the households include at least one employed individual, although, this data

\footnotetext{
8 The $t$-statistic is calculated according to the formula

$t=\frac{P_{A}-P_{B}}{s}$ where $s=\sqrt{\operatorname{var}\left(P_{A}\right)+\operatorname{var}\left(P_{B}\right)}$. Further details can be found in Ravallion and Datt (1996) and Kakwani (1993).

${ }^{9}$ Note that we are effectively including the pregnant women in the distribution of the number of employed within the households. Hence, the number of employed includes these women, whether they are working or not. We turn in the next section to individual characteristics to shed more light on their labour market status within the sample.
} 
does not tell us about the quality of this support in the form of monetary value of the wages earned.

Table 6. Distribution of Employed Across Households by Imputed HIV Status

\begin{tabular}{llll}
\hline Number of Employed & HIV Negative & HIV Positive & Total \\
0 & 44.5 & 42.2 & 43.5 \\
1 & 31.3 & 29.9 & 30.8 \\
2 & 13.6 & 16.2 & 14.8 \\
$3+$ & 10.5 & 11.5 & 10.7 \\
Total Share & 100 & 100 & 100 \\
Estimated Statistics & & & \\
Mean & 0.966 & 1.036 & 1.000 \\
Std. Error & 0.036 & 0.0717 & 0.0297 \\
\hline
\end{tabular}

Source: OHS99 and authors' own calculations

In the case of imputed HIV negative women, approximately 55 per cent of these household types had access to at least one wage-earner, compared with some 58 per cent of HIV positive women. At face value then, the imputed HIV positive women appeared to have access to more income earners than imputed HIV negative women. This fact is borne out by the estimated mean figures, where the imputed HIV negative mean was marginally lower (0.966) than that for the imputed HIV positive sample (1.036). Ostensibly then, this evidence may appear to contradict the initial cumulative distribution functions, which suggested that imputed HIV positive women came from poorer households - given that they appeared to have access to a greater number of wage earners within a household.

There is one important caveat to these results: more women from the HIV positive cohort were located in households with more (or the same number of) earners, but the quality of the support offered could be lower. Put differently, while the number of employees in the household may be larger, the aggregate value of income earned by these individuals might be smaller for imputed HIV negative households as shown in the initial analysis that examined cumulative income distributions and poverty measures for the full sample (Fig. 1 and Table 6). Again, the small sample size problem may render this analysis highly questionable. However, we undertook it to at least indicate the linkages that can and should be made to understand household attachment patterns amongst HIV afflicted individuals.

The cumulative distribution functions of imputed HIV status households are produced below, with the first figure examining the distribution for those households with one or more earners (Fig. 2a), and the second figure illustrating the comparative distributions for households with zero earners (Fig. 2b).

The results are instructive, and reflect on the importance of linking 'quantity of support' in terms of number of earners to 'quality of earners' in the form of the value of income entering the household. It is clear, at least graphically, from Fig. 2a that apart from the lowest income band, the proportion of imputed HIV positive households earning below any given income band is greater than the imputed HIV negative cohort. For example the Fig. shows that while 60 per cent of HIV negative households earned below R1199 per month, 68 per cent of imputed positive households earned below this figure. This suggests that while the quantity of support available to imputed HIV positive households may be greater (or the same as) imputed HIV negative households, the quality of support in the form of monetary value is different. 


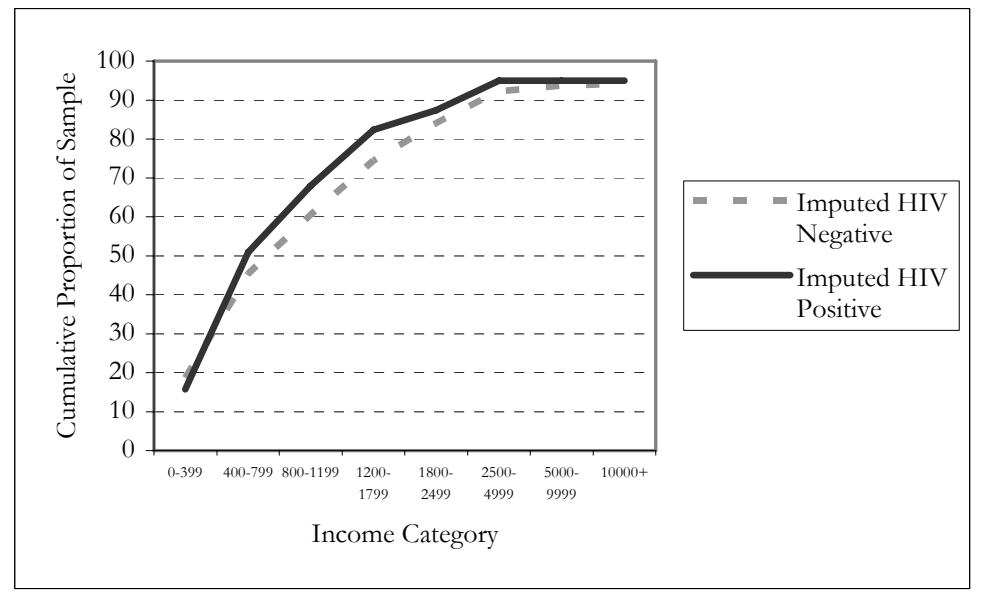

Figure 2a. Cumulative Distribution of Monthly Household Income for Households with at Least One Wage Earner, by Imputed HIV Status

Source: OHS99 and authors' own calculations

We examined in turn, in the Fig. $2 \mathrm{~b}$ below, the cumulative distribution functions for those households who reported having zero wage income earners. Fig. 2a suggested that the imputed HIV positive cohort had a lower proportion of these households. Fig. $2 \mathrm{~b}$ shows though, the quality of the support offered differed markedly.

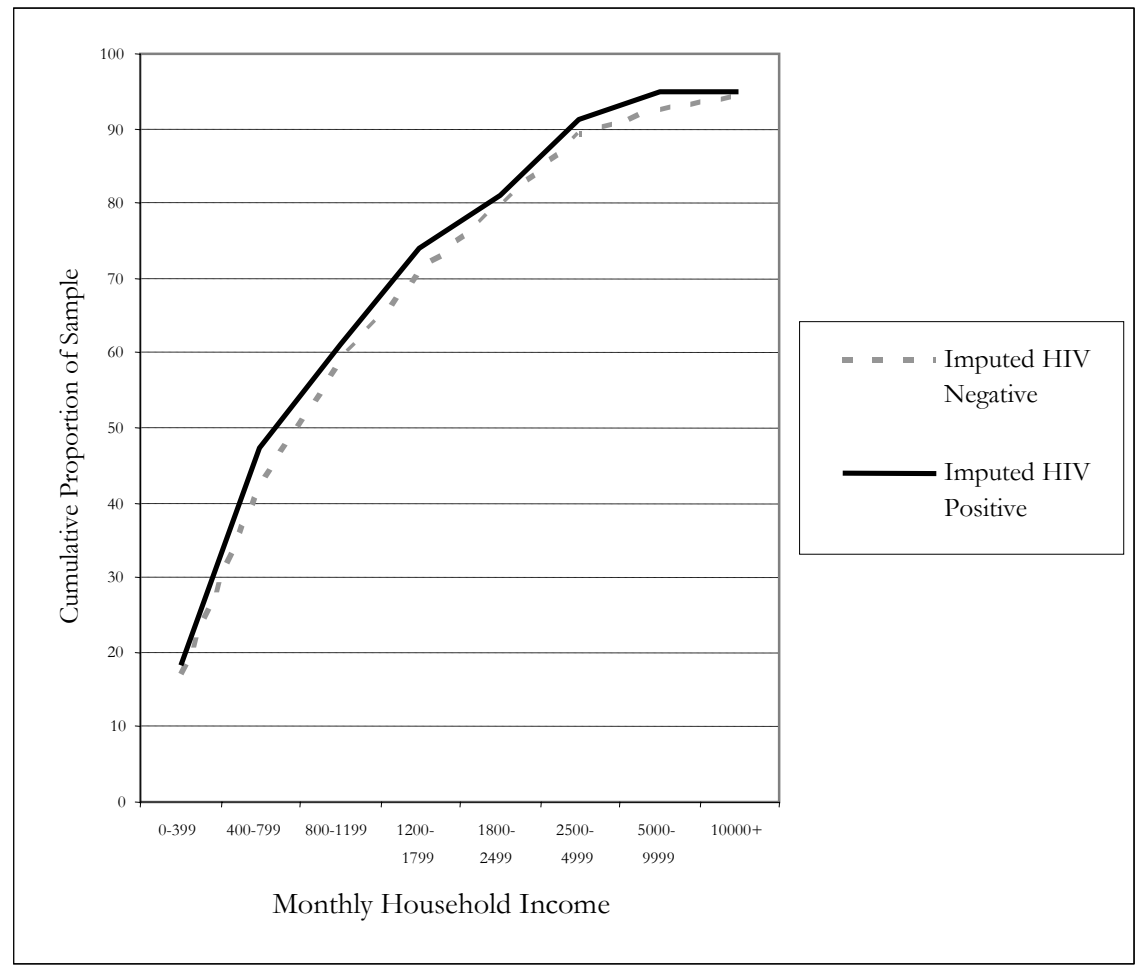

Figure 2b. Cumulative Distribution of Monthly Household Income for Households with at Least One Wage Earner, by Imputed HIV Status

Source: OHS99 and authors' own calculations 
We found that for every income category, the cumulative function for imputed HIV positive households was above that of imputed HIV negative households. Put differently, across all household income bands, first order dominance holds - with the HIV positive distribution first-order dominating the HIV negative distribution. For example, within the 1200-1799 per month income range, the proportion of imputed HIV negative households below this range is 70.61 per cent, compared to 74.14 per cent for imputed HIV positive households. In addition, within the range R400-799, the proportion below this is 42.24 per cent for imputed HIV negative households and 47.40 per cent for imputed HIV positive households.

Next, we attempted to provide point estimates of vulnerability through the estimation of the headcount and poverty gap measures for the two samples. As with the above estimation, we retained the poverty line of R1499.5 per month. The results in Table 7 present the poverty measures for the imputed HIV positive and negative women who lived in households with at least one wage earner. The results for those with zero wage earners are provided in the appendix below. Both the headcount and poverty gap measures were higher for imputed HIV positive households. In terms of the latter, while imputed HIV positive households were on average 46 per cent below the poverty line, the figure for imputed HIV negative households was 44 per cent. Put differently, imputed HIV positive households were relatively more poor than imputed HIV negative households, where both had at least one wage earner residing.

Table 7. Headcount and Poverty Gap Measures by Imputed HIV Status Households with at least One Wage Earner

\begin{tabular}{lllll}
\hline $\begin{array}{l}\text { Poverty Measure } \\
\text { HIV Negative }\end{array}$ & Estimate & Std. Error & Confidence Interval & \\
Headcount Index & 0.67 & 0.019 & 0.6281 & 0.7030 \\
Poverty Gap & 0.44 & 0.014 & 0.4070 & 0.4637 \\
HIV Positive & & & & \\
Headcount Index & 0.73 & 0.035 & 0.6618 & 0.8007 \\
Poverty Gap & 0.46 & 0.026 & 0.4064 & 0.5097 \\
t-Statistics & Headcount & $-1.64 * *$ & Poverty Gap & -0.76 \\
\hline
\end{tabular}

Source: OHS99 and authors' own calculations

Note: Poverty Line z, is set at 1499.5 per month per household.

**: Significant at the 10 per cent level.

The headcount measure is significant at the 10 per cent level, while the difference in the poverty gap estimates was insignificant. The analysis which lead to the figures shown in Table 7 was repeated for households with no wage earners, at the same poverty line. Here again the headcount and poverty gap were higher for imputed HIV positive households. However, for both measures, the $t$-statistic was insignificant.

Finally, sensitivity tests for a raised poverty line of R2149.5 per household per month, the midpoint of the range 1800 to 2499, indicated the same broad trends, with the poverty estimates for imputed HIV positive higher than those for the imputed HIV negative households Notably, the result was significant for differences in the headcount across $1+$ wage earner households.

These results are an important extension to our initial finding which was that imputed HIV positive women appear to have been residing in poorer homes than imputed HIV negative women. They suggest two key further features of the vulnerability profile of the pandemic. Firstly, imputed HIV positive and negative 
cohorts were located in similar proportions, within wage-earning households. Secondly, the access to wage earners within households with imputed HIV positive women implied that location was not optimal, given that they resided in households with lower levels of income. The households with either no wage earners or at least one, were poorer among HIV positive women than HIV negative women. Put differently, the labour market participants that positive women were accessing within the households are likely to be more vulnerable than those accessed by imputed HIV negative women. Finally, it is important to emphasize that these results are tentative, given the small differences in poverty. However, we do believe that the lack of significance in the $t$ tests is driven by the smallness of the sample size, and that the direction and nature of the differences would be likely to remain, should a larger sample size be utilized.

\section{(d) Attachment to the Unemployed and Non-Wage Earners}

As an extension to the above, it would be useful to determine the nature of intrahousehold dynamics in the form of the access to non-wage earners, such as pensioners, that this sample displays. In addition, it is important to gauge the burden placed on these households by the unemployed. We turn to the latter in the table below, which examines the distribution of the unemployed across the two household types. It is evident firstly, that almost all households in the sample report the presence of an unemployed individual ${ }^{10}$. In the case of the imputed HIV positive sample, there are no households with zero unemployed individuals in them.

About 63 per cent of both cohorts reported between one and four unemployed household members with the distribution peaking in households with three unemployed individuals, for both groups.

\section{Table 8. Distribution of Unemployed Across Households by Imputed HIV Status}

\begin{tabular}{llll}
\hline Number of Unemployed & HIV Negative & HIV Positive & Total \\
0 & 0.09 & 0.00 & 0.06 \\
1 & 3.44 & 3.96 & 4.24 \\
2 & 15.75 & 16.97 & 16.21 \\
3 & 23.53 & 24.55 & 22.78 \\
4 & 19.91 & 17.33 & 19.17 \\
$5+$ & 37.28 & 37.19 & 37.54 \\
Total & 100 & 100 & 100 \\
Estimated Statistics & & & \\
Mean & 4.327 & 4.137 & 4.248 \\
Std. Error & 0.0746 & 0.1181 & 0.057 \\
\hline SOurce: OHSOS
\end{tabular}

Source: OHS99 and authors' own calculations

The mean for both samples is just over four unemployed individuals per household. In the case of imputed HIV positive households, it is in fact slightly lower at 4.137 as opposed to 4.327 for imputed HIV negative households. Again though, it was important to determine whether the levels of income across these household types were different. In other words, while the distribution of the unemployed across the two cohorts may be similar, imputed HIV positive women may still emanate from poorer homes. The headcount and poverty gap estimates for imputed HIV positive and negative households with at least one unemployed member, bear this out. The

\footnotetext{
10 As the section below will elucidate on, we use the expanded definition of unemployment here to differentiate between the unemployed, employed and economically inactive.
} 
headcount index for imputed HIV positive women (70 per cent) is at least four percentage points higher than that for imputed HIV negative women (66 per cent); and there is a two percentage point difference in the poverty gap measure (45 per cent versus 43 per cent). This reiterates our two earlier results, namely that imputed HIV positive women are residing in poorer households - a result that we would of course expect given that we are essentially replicating the sample of women in the aggregate figures presented above.

It has been shown that internationally income transfers remain effective instruments for poverty alleviation. South Africa is no exception, and the old age pension in particular has been shown to be a highly optimal transfer in terms of reaching poor households. In Table 9, we examine whether access to the old age pension, and another important income transfer - remittances - differs across these two household categories $^{11}$.

Table 9. Presence of Non-Wage Earner(s) in Household by Imputed HIV Status Households (\%)

\begin{tabular}{llll}
\hline Pensioner(s) Present & HIV Negative & HIV Positive & Total \\
Pensioner Present? & & & 80.23 \\
No & 80.9 & 78.7 & 19.77 \\
Yes & 19.1 & 21.3 & 100 \\
Total & 100 & 100 & \\
Estimated Statistics & & & 0.2344 \\
Mean & 0.2253 & 0.2599 & 0.0125 \\
Std. Error & 0.0151 & 0.0322 & \\
Remitter Present? & & & Total \\
Remitter(s) & Negative & Positive & 84.69 \\
No & 84.44 & 86.28 & 15.31 \\
Yes & 15.56 & 13.72 & 100 \\
& 100 & 100 & 0.1886 \\
Estimated Statistics & & & 0.0134 \\
Mean & 0.1855 & 0.1877 & \\
Std. Error & 0.0146 & 0.0399 & Total \\
Any Non-Wage Earner Present? & & & 29.97 \\
No. of non-wage income earners & Negative & Positive & 70.03 \\
No & 30.68 & 29.96 & 100 \\
Yes & 69.32 & 70.04 & 1.42 \\
Total & 100 & 100 & 0.0352 \\
Estimated Statistics & & & \\
Mean & 1.3819 & 0.0898 & \\
Std. Error & 0.04231 & &
\end{tabular}

Source: OHS99 and authors' own calculations

Firstly, in the case of both old age pensions and remittances, the majority of households in both imputed HIV categories reported having no access to these transfers. About 80 per cent of all households in the sample have no old age pensioner living with them, and about 85 per cent of all households have no recipient of remittances. Hence, households appear to have minimal access to these two major income transfers. For this reason, we investigated household access to any form of income transfer. In

11 We have excluded other income transfers such as the child support grant, disability grant and unemployment insurance, given that a very small proportion of the sample in either of the two categories reported having access to these transfers. For example, in the case of the disability grant, between 95 and 97 per cent of households in each of the two categories reported having no access to this grant. 
addition to the two already noted, these transfers include disability grants, the child support grant, unemployment insurance, care dependency grant and so on. When every form of income transfer was considered, the level of support provided clearly increases; between 69 (imputed HIV negative) and 70 per cent (imputed HIV positive) of households report accessing at least one form of these transfers.

The estimated means revealed that across both transfer types and the category covering all grants, imputed HIV positive households have access to a marginally larger number of these recipients; for example, the mean number of old age pensioners in imputed HIV positive households was 0.26 , compared to 0.23 for imputed HIV negative households. These differences suggest that imputed HIV positive and negative households are accessing the same quantum of income transfer recipients.

Predictably, the next question is whether access to the same number of recipients enabled imputed HIV positive households, to be less poor, or at least not poorer, than imputed HIV negative households? The poverty measures for those households with at least one non-wage income earner resident, by imputed HIV status, are shown in Table 10. It is evident that through consideration of both the headcount and poverty gap measures, imputed HIV positive households are poorer. Hence, access to the same number of income transfers was not sufficient to make imputed HIV positive households less or as poor as imputed HIV negative households.

Table 10. Headcount and poverty gap measures for imputed HIV status households with at least one non-wage income earner

\begin{tabular}{lllll}
\hline $\begin{array}{l}\text { Poverty Measure } \\
\text { HIV Negative }\end{array}$ & Estimate & Std. Error & Confidence Interval & \\
Headcount Index & 0.67 & 0.017 & 0.6363 & 0.7031 \\
Poverty Gap & 0.43 & 0.013 & 0.4050 & 0.4549 \\
HIV Positive & & & & \\
Headcount Index & 0.74 & 0.031 & 0.6802 & 0.8044 \\
Poverty Gap & 0.47 & 0.024 & 0.4209 & 0.5141 \\
t-Statistics & Headcount & $-2.03^{* *}$ & Poverty Gap & -1.40 \\
\hline
\end{tabular}

Source: OHS99 and authors' own calculations

Note: Poverty Line z, is set at 1499.5 per month per household.

**: $\quad$ Significant at the 10 per cent level.

The absolute measure was statistically significant at the 10 per cent level, indicating a robust measure of poverty differences between the two samples of pregnant women, while the relative measure of poverty was insignificant. Ultimately though, this suggests that income transfers in and of themselves are, at least in this small sample, not able to lift a greater proportion of imputed HIV positive households out of poverty, relative to imputed HIV negative households.

The above data raises an important question concerning intra-household dynamics that may be resulting in these skewed poverty outcomes. Despite having ostensibly the same access levels to both wage and non-wage earners and yielding similar household sizes, together with similar dependency ratios, imputed HIV positive households seemed to be consistently poorer than imputed HIV negative households. We turn now to the individual characteristics of the sample, in an attempt to deal in greater detail with this issue. 


\section{INDIVIDUAL CHARACTERISTICS BY IMPUTED HIV STATUS}

Having dealt with the characteristics of the households wherein these two cohorts of pregnant women resided, it was important to assess how their individual characteristics differed across a range of covariates. In the following sections, we cover the differing labour market statuses of these individuals, including wages earned, their marital status, relationship to the head of the household and so on. The broad aim of this section is to understand what specific individual characteristics by imputed HIV status may possibly be shaping the differential household outcomes observed above.

\section{(a) Labour Market Characteristics by Imputed HIV Status}

It is possible that the differential household poverty levels amongst imputed HIV positive and negative women is in fact driven by the nature of their labour market status. Table 11 details the respective labour market statuses according to both the strict (or official) definition of unemployment and the expanded definition. We report in each case the share of women within each labour market category, namely; not economically active (out of the labour force), employed and unemployed. As a derivation of these, we also report the unemployment rates for the two groups.

According to either definition, the share of imputed HIV positive women out of the labour force is marginally lower, with 64 and 41 per cent not economically active, respectively.

Table 11. Labour market status by imputed HIV status (OHS99)

\begin{tabular}{|c|c|c|c|}
\hline Labour Market Status & Negative & Positive & Total \\
\hline \multicolumn{4}{|l|}{ Official Definition } \\
\hline Not Economically Active & 68.3 & 64.49 & 67.65 \\
\hline Employed & 18.12 & 15.58 & 16.96 \\
\hline Unemployed & 13.59 & 19.93 & 15.39 \\
\hline Total & 100 & 100 & 100 \\
\hline \multicolumn{4}{|l|}{ Estimated Statistics } \\
\hline Unemployment rate & $30.00(1.297)$ & $35.95(1.993)$ & $32.24(0.986)$ \\
\hline \multicolumn{4}{|l|}{ Expanded Definition } \\
\hline Not Economically Active & 43.3 & 40.94 & 43.39 \\
\hline Employed & 18.12 & 15.58 & 16.96 \\
\hline Unemployed & 38.59 & 43.48 & 39.65 \\
\hline Total & 100 & 100 & 100 \\
\hline \multicolumn{4}{|l|}{ Estimated Statistics } \\
\hline Unemployment rate & $40.49(0.660)$ & $42.40(1.118)$ & $41.19(0.514)$ \\
\hline
\end{tabular}

In terms of active participants though, it is evident that the share of women who are employed is greater for imputed HIV negative women (18 per cent) compared with imputed HIV positive women (16 per cent). Hence, this is an early suggestion that imputed HIV positive women are less likely to be in employment (assuming they are in the labour force) than imputed HIV negative women. This differential share in employment is of course a reflection of the contrast in access to wage income, a vital component of household income. These employment shares are then masked in the differing unemployment rates across the two groups, which we provide in estimated form with their standard errors in the Table 11. It is evident that the strict unemployment rate for imputed HIV positive women is 36 per cent, while for the 
negative group it is six percentage points lower. In terms of the expanded definition, the unemployment rate is 42 per cent for imputed HIV positive, and 40 per cent for imputed HIV negative women. Provisionally, this suggests that, by both definitions of unemployment, imputed HIV positive women are more likely than imputed negative women to be unemployed, and therefore face a higher likelihood of being zero earners. In this manner the labour market enters as one of the key, if not the key, factor in understanding the differential household poverty levels noted in the previous section. The fact that imputed-positive women are more likely to be unemployed, serves as one of the primary mechanisms for ensuring that the households they reside in are poorer than those households with imputed HIV negative women.

Having established that there is a lower probability of employment amongst the imputed negative cohort, it is also relevant to determine the characteristics of unemployed women across both groups. We examined employment by a series of covariates for these two samples to assess whether these figures reinforce the initial result of differing levels of vulnerability for the two groups. The weekly wages by imputed HIV status, for those women who are employed are examined in Fig. 3 or 2? below $^{12}$. We have presented the data according to percentiles in the respective wage distributions, ranging from the $5^{\text {th }}$ to the $95^{\text {th }}$ percentile for each of the distributions. The figures are particularly revealing, in that they show distinct patterns in the distributions.
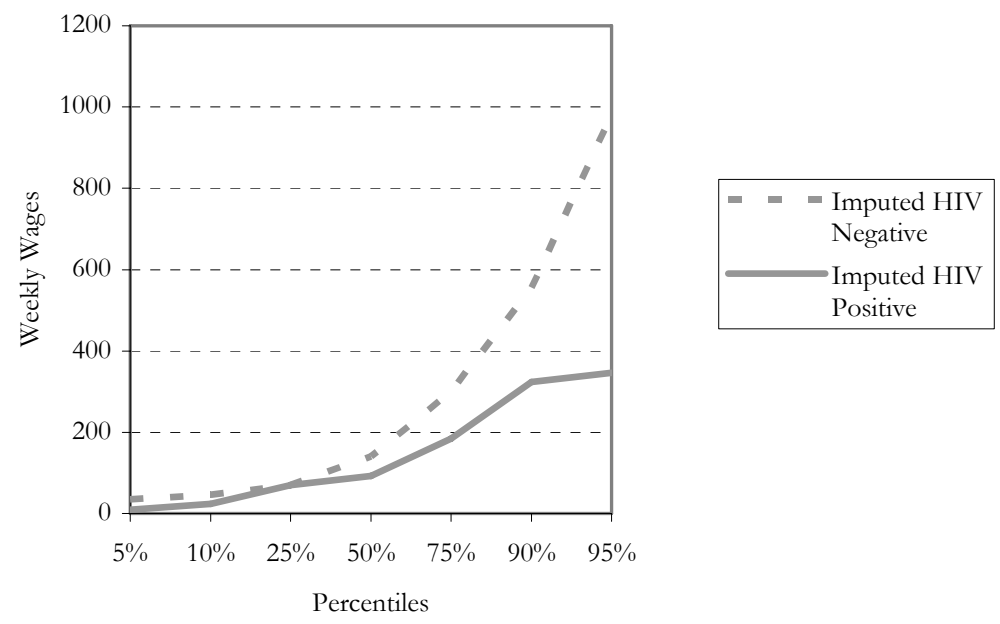

Figure 3. Percentile Distribution of Weekly Wages, by Imputed HIV Status

Source: OHS99 and authors' own calculations

In the bottom-quarter of the wage distribution $\left(5^{\text {th }}-25^{\text {th }}\right.$ percentile), it is evident that weekly wages are fairly closely correlated relative to the distribution from the $50^{\text {th }}-95^{\text {th }}$

12 The OHS99 does have a question on actual wages earned, but respondents can provide it in any frequency format (weekly, monthly or annual), which is specified in the questionnaire. Hence, it was necessary to convert all monthly and annual wages to their weekly equivalent. This was done using a factor of 0.231 for monthly wages and a factor of 0.0192 for annual wages. 
percentiles. Throughout the distribution however, the weekly wages of imputed HIV positive women is below that of imputed HIV negative women. For example, the $10^{\text {th }}$ percentile wage is R32.10 per week for positive women while it is R46.20 for negative women. Even at the median (the $50^{\text {th }}$ percentile), the weekly wage of imputed positive women is lower at R92.40 compared with R140 for imputed negative women. Despite this differential in the bottom half of the distribution, it is clear though that it is in the upper portions of the distribution, that the differentials begin to widen even further. Data on the differing ratios across the percentiles indicates that the ratio of HIV negative to HIV positive wages declines steadily to the $50^{\text {th }}$ percentile, and then increases thereafter. For example, while at the $50^{\text {th }}$ percentile the weekly wage of imputed negative women is about 150 per cent of the same percentile wage for imputed positive women, at the $90^{\text {th }}\left(95^{\text {th }}\right)$ percentile, this ratio falls to 171 (282 per cent). There would appear then to be two key deductions from the wage data above. Firstly, that imputed positive women who are employed are earning lower weekly wages than those who are imputed-negative. Secondly, this differential tends to decline steadily until the median of the distribution, after which the differentials begin to widen again.

Mindful of our small sample size, the above provides at least tentative evidence for a second labour market factor affecting the contrasting household poverty levels. Not only are imputed HIV positive women more likely to be unemployed, but the evidence here points to the fact that should they be employed, they are likely to earn a wage that is lower than their counterparts who are imputed HIV negative.

In trying to understand what factors may be shaping these relatively lower earnings amongst imputed HIV positive women, we tabulate in Table 12 the distribution of employment amongst the two cohorts by sector, occupation and level of formalisation. Beginning with the latter, the data below suggests that the majority of women in this sample are employed in the formal sector.

Table 12. Formal-Informal Sector Distribution of the Employed by Imputed HIV Status

\begin{tabular}{llll}
\hline Sector & Negative & Positive & Total \\
Formal & 75.8 & 58.33 & 72.37 \\
Informal & 24.2 & 41.67 & 27.63 \\
Total & 100 & 100 & 100 \\
\hline
\end{tabular}

Source: OHS99 and authors' own calculations

However, the imputed positive women are more likely to be in the informal sector than imputed negative women. This would partly explain the lower wages earned by imputed HIV positive women in the figure above. Indeed, more detailed evidence shows the mean wage of imputed positive women in the informal sector is about 60 per cent of the wage earned by imputed negative women in the formal sector. In addition, the mean wage within the formal sector is higher for imputed negative women, when compared with the imputed positive sample. Examining the relationship of sectoral distribution by HIV status through a Chi square analysis for categorical data, we find a significant relationship between being employed in the informal sector and having a positive HIV status $(\mathrm{p}=0.03)$. The Relative Risk of women being HIV positive if they were employed in the informal sector versus the formal sector was 1.89 (CI 95\%: 1.053.38).

In trying to expand on the role of the labour market in engendering these household poverty outcomes, we further examined the distribution of employment by sector and occupation for these two cohorts Table 13 indicates that the dominant sectors for 
imputed negative women are Wholesale and Retail Trade and Private Households. The second of these captures, in the main, those women employed as domestic workers.

Table 13. Sectoral Distribution of the Employed by Imputed HIV Status (OHS99)

\begin{tabular}{llll}
\hline Sector & Negative & Positive & Total \\
Agriculture & 15.5 & 13.95 & 14.23 \\
Manufacturing & 12.5 & 11.63 & 12.1 \\
Construction & 0.00 & 2.33 & 0.36 \\
Wholesale and Retail Trade & 34.5 & 20.93 & 32.74 \\
Transport, Storage, Communication & 0.00 & 2.33 & 0.36 \\
Finance & 4.50 & 6.98 & 4.63 \\
Community, Social and Personal Services & 16.00 & 13.95 & 15.66 \\
Private Households & 16.00 & 25.58 & 18.86 \\
Not Specified & 1.00 & 2.33 & 1.07 \\
Total & 100 & 100 & 100 \\
\hline Source: OHSTS
\end{tabular}

Source: OHS99 and authors' own calculations

It is clear also that Agriculture and Community Services, constitute over a quarter of the sample of employed imputed positive women. The latter sector is predominantly represented by the public sector.

The dominance of both the above sectors is not surprising, given that these sectors are large employers in the national economy, and tend also to employ larger shares of women than other sectors such as Mining and Financial and Business Services. These figures alone do not adequately explain the differential levels of household vulnerability, although they certainly suggest employment distributions that are distinct across the two cohorts. Marked differentials exist among women working in private households by HIV status, namely a smaller proportion of HIV negative women are employed at the private household level than HIV positive $(16 \% \text { versus } 26 \%)^{13}$.

Ultimately though, several important corollaries match well with the empirical description of significant differences in household vulnerability between these two groups of pregnant women. First, it is evident that one of the key factors driving this differential household poverty status, is the fact that imputed positive women are less likely than imputed negative women to find employment in the labour market. The estimated unemployment rates confirm this difference in employment probabilities across the two groups. Secondly, for those women that are employed, there is initial (although insignificant) evidence that their earnings are lower if they have an imputed HIV positive status. This evidence is then corroborated by the sectoral distributions, which reveal that employed HIV positive women are more likely to be employed as household domestic workers and less likely to be working in the higher-paying formal sector than their imputed-HIV positive counterparts. Collectively these data strongly suggest that the differential individual labour market characteristics of imputed HIV positive women is negatively influencing their household poverty status, relative to the imputed HIV negative cohort.

\footnotetext{
13 Note that we did attempt to run a table on the contrasting employment distributions by occupation across the two cohorts. However, close to 20 per cent of the sample of imputed negative women and a quarter of imputed positive women reported an unspecified occupation, rendering this data unusable.
} 


\section{CONCLUSIONS}

This study aimed to provide a tentative analysis of the relationship between HIV, poverty and labour markets. It is clear from the paper that the relationship between poverty, labour markets and HIV is not homogenous but multi-dimensional in character. The analysis examined these inter-relationships at both the household and individual level. The key findings from the analysis suggest that imputed HIV positive women come from poorer households than imputed negative women.

The relationship between imputed HIV status and the various descriptors of poverty and labour market status is palpable and apparent at the household level. The household level analysis revealed that that there is a differential access to the level of income across imputed HIV status in this sample of pregnant women. More detailed analysis gauging poverty through the use of indices such as the headcount ratio and the poverty gap showed that the imputed HIV positive cohort was significantly different from the non-infected group in that the former resided in poorer households. This is a critical result as it provides initial evidence suggesting that imputed HIV positive households are worse off than the imputed HIV negative households in terms of both absolute and relative poverty levels.

An important household welfare indicator is an individual's attachment to wage earners, whereby the degree of support can be gauged by their access to wage earners. This analysis revealed that imputed HIV positive women appear to have access to more income earners than imputed HIV negative women even though they reside in poorer households. However, when we further examine the quality of the support of the wage earners, we find that the level of support to imputed positive women is lower. Thus, the results indicate that households with at least one wage earner, are poorer in the case of HIV positive women, than HIV negative women.

In the case of access to non-wage earners, such as pensioners we find that the quantity of support provided to imputed HIV positive women is greater than for imputed negative women. However, in trying to measure the quality of support, the poverty measures for households with at least one non-wage income earner resident shows that for the headcount and poverty gap indices, imputed HIV positive households are poorer. These findings provisionally suggest that income transfers are not sufficient to lift a greater proportion of imputed HIV positive households out of poverty, relative to imputed HIV negative households. Despite having ostensibly the same access to both wage earners, and increased access to non-wage earners (in households with similar dependency ratios), imputed HIV positive households seem to be consistently poorer than imputed HIV negative households.

Individual characteristics by imputed HIV status were examined to further understand why HIV positive women come from poorer homes. The key findings include the composite observation that women who are most affected by the HIV epidemic are essentially young (aged 20-29), living in urban settings and unemployed. These findings are consistent with a national household survey that examined sociobehavioural factors in relation to HIV infection in South Africa (Shisana and Simbayi, 2002). Higher HIV rates in urban settings have been attributed to combinations of factors such as rapid urbanization, migration, unemployment and unstable families or communities (Quinn, 1996).

The study revealed that by both definitions of unemployment, imputed HIV positive women are more likely than imputed-negative women to be unemployed. In addition, 
the imputed-positive women who are employed are also earning lower weekly wages than those who are imputed-negative with the wage differentials tending to be very wide at the tail-ends of the distributions.

The wage differentials are supported by further analysis that examined the sector of employment by HIV status. Imputed positive women are more likely to be employed in the informal sector than imputed negative women. In addition, the mean wage within both the formal and informal sector is higher for imputed negative women, when compared with the imputed positive sample. The high proportion of women in the Retail and Private Household (domestic worker) sectors is consistent with national trends as these sectors are either larger employers or disproportionate employers of women.

The employment profile suggests that imputed positive women are more likely than imputed negative women to be unemployed, and if employed, they are likely to be employed at the bottom-end of the occupational ladder, with contrasting lower wages. This profile validates the empirical description of significant differences in household vulnerability between these two groups of pregnant women. The key appears to be employment status of the imputed HIV positive women, which then has a deleterious impact on their household poverty status, relative to the imputed HIV negative cohort.

In South Africa, there is a dearth of national level information on the relationship between HIV and poverty, and the implications for the labour market. However, there is substantial work on modelling and projecting the epidemic using the HIV antenatal survey data and on a more limited scale, some localized analysis of the relationship between poverty and HIV at household level. This study presents an innovative method of using available data, namely the ANC and OHS data, to simulate the impact of HIV on households and individuals with respect to poverty and labour markets. This analysis provides an initial window into how the HIV epidemic affects young pregnant women attending public sector clinics. Examining the impact of the epidemic in this group is significant as they represent young sexually active adults, who form the foundation of the current and future labour force. This analysis consistently showed through individual and household level analysis that women who are imputed HIV positive are poorer than the imputed HIV negative group. Whilst the absolute magnitude and severity of poverty and HIV cannot be measured (nor can the causality be tested with the current available data\}, they provide valuable insights into the relationship between these factors. They also provide evidence that at both policy and programme implementation levels, the HIV epidemic cannot be managed without systematic and rigorous efforts that address poverty at individual and household levels.

\section{REFERENCES}

AWOFESO, N., (1995). AIDS and tuberculosis/leprosy in Nigeria: the urbanization factor. Acta Leprol. 9(3): 149-51. BACHMANN, M., BOOYSEN, F., (2003). Health economic impact of HIV/AIDS on South African households: A cohort study. BMC Public Health. 3(1):3-14.

DePartment OF HeAlth, (1998). 1998 National HIV Sero-Prevalence Survey of Women Attending Public Health Antenatal Clinics in South Africa. 1-11. Department of Health, Pretoria.

(2000). National HIV Sero-Prevalence Survey of Women Attending Public Health Antenatal Clinics in South Africa. 1-14 . Department of Health, Pretoria.

FOSTER, J.E., GREER, J. AND THORBECKE, E., (1984). “A Class of Decomposable Poverty Measures”. Econometrica.52 KAKWANI, N., (1993). "Statistical Inference in the Measurement of Poverty". The Review of Economics and Statistics 75(4). MONITORING THE AIDS PANDEMIC (MAP) NETWORK (1998). Status and trends of HIV / AIDS epidemics in the World. UNAIDS (Joint United Nations Programme on HIV/AIDS.

NICOLl, A., GILl, ON., PECKHAM, CS., ADES, AE., PARry, J., MORTIMER, P., GOldBERG. D., NOONE, A., BENNETT, D., 
CATCHPOLE, M., (2000). The public health applications of unlinked anonymous seroprevalence monitoring for HIV in the United Kingdom Int Journ Epi. 29: 1-10.

QUINN, TC.,(1996). Global burden of the HIV pandemic Lancet. Jul 13; 348(9020): 99-106.

RAVAllion, M., AND DATT, G., (1996). Is Targeting through a Work Requirement Efficient? Some Evidence for Rural India. In: D. van der Walle \& K. Nead (eds) Public Spending and the Poor: Theory and Evidence. The Johns Hopkins University Press. Baltimore, Maryland.

SHAikH, N., AND ABDUllaH, MF., (2002). The Provincial and District Survey Report Western Cape 2002. ISBN 0-62027758-0, 1-23. Cape Town

SHISANA, O., SIMBAYI, L., (2002). Nelson Mandela /HSRC study of HIV/AIDS .South African National HIV prevalence: Behavioral Risks and Mass Media. Human Sciences Research Council. ISBN 0-7969-2007-9; 58-59. Cape Town.

TOPOUZIS, D., (1998) The implications for HIV/AIDS for rural development policy and programming: Focus on sub-Saharan Africa, Sustainable Development Department UNDP and FAO, UNDP, Geneva

WORLD HEALTH ORGANISATION. (2000). WHO report on Global Surveillance of Epidemic - Prone Infectious Diseases. HIV and AIDS ,Pg 18.Geneva

(1989). Unlinked anonymous screening for public health surveillance of HIV infections. Proposed International guidelines. Global Programme on AIDS, GPA/SFI/89.3:1-7. Geneva. 